\title{
Challenges of Critical Cardiac Imaging in Cardiogenic Shock
}

\author{
Marius George Linguraru* \\ Sheikh Zayed Institute for Pediatric Surgical Innovation, Children's National Health System, Washington DC, USA
}

Received: 15 June 2015 / Accepted: 26 June 2015

\section{-TO THE EDITOR OF JCCM}

I am writing in reference to the article published by Theodora Benedek and Dan Dobreanu in the first issue of JCCM, entitled "Current Concepts and New Trends in the Treatment of Cardiogenic Shock Complicating

Acute Myocardial Infarction".

Cardiogenic shock (CS) represents a critical and life-threatening condition. Survival of patients with CS depends largely, not only on the appropriateness of the therapeutic measures, but also on the correct identification of the underlying disease [1].

Treatment of this underlying condition represents a key element for the correction of the patho-phyisiological pathways responsible for the development of a CS.

In many cases, $C S$ occurs in association with an acute myocardial infarction, usually large infarcts, located on the anterior ventricular wall [2]. Prompt revascularisation is crucial in these cases, as the re-establishment of coronary flow would immediately improve the haemodynamic status of these critically ill patients. However, in routine clinical practice, the diagnosis of an acute coronary syndrome remains challenging, especially when the physician is faced with a patient who arrives in the emergency room (ER) intubated, after surviving a cardiac arrest of unknown aetiology. In these cases, it is of extreme importance to differentiate between other possible causes of CS, such as pulmonary embolism or acute aortic dissection. Cardiac imaging plays a critical role in diagnosing patients with critical cardiac conditions.

The "triple rule-out" exam has been proposed for Cardiac Computed Tomography (CCT) in the ER. This examination allows to rule-out the three major causes of cardiogenic shock: acute coronary syndromes, pulmonary embolism and acute aortic dissection [3]. While CCT examination has been shown to be efficient for the vast majority of patients presenting in the ER with a chest pain, CCT imaging a patient with cardiac arrest or CS is not a trivial task.

The above mentioned article published recently in the JCCM discussed the treatment options available nowadays for CS cases, emphasizing the critical role of new devices for providing mechanical circulatory support [4]. However, the addition of a circulatory device makes cardiac imaging even more technically challenging. For example, even the simple use of the classical balloon counter-pulsation pump may not only make the handling of the patient more difficult, but could also further impact on the quality of the obtained image.

In conclusion, the role of cardiac imaging in the ER is well established, and several randomised controlled trials reported the advantages of imaging examinations for the evaluation of patients with acute chest pain [5]. However, challenges of CCT in the assessment of patients presenting with CS or after surviving a cardiac arrest requires further investigations. A combined approach, incorporating CCT imaging and analysis together with determination of serum levels of highsensitive troponins could potentially provide a valuable diagnostic system for the ER.

\section{CONFLICT OF INTEREST}

None

\section{REFERENCES}

1. Hochman JS. Cardiogenic Shock Complicating Acute Myocardial Infarction- Expnding the paradigm. Circulation. 2003;107:29983002 . 
Available online at: www.jccm.ro

2. Szymanski FM, Filipiak KJ. Cardiogenic shock - diagnostic and therapeutic options in the light of new scientific data. Anaesthesiol Intensive Ther. 2014;46:301-6.

3. Ropp A, Lin CT, White CS. Coronary computed tomography angiography for the assessment of acute chest pain in the emergency department: evidence, guidelines, and tips for
The Journal of Critical Care Medicine 2015;1(3) • 119

implementation. J Thorac Imaging. 2015;30:169-75.

4. Benedek T, Dobreanu D. Current Concepts and New Trends in the Treatment of Cardiogenic Shock Complicating Acute Myocardial Infarction. J Crit Care Med. 2015;1:5-10.

5. Nieman K, Hoffmann U. Cardiac computed tomography in patients with acute chest pain. Eur Heart J. 2015;36:906-14. 